\title{
INTELLIGENT DESIGN OF VEHICLE PACKAGE USING ONTOLOGY AND CASE- BASED REASONING
}

\author{
Xiaoping Jin ${ }^{1}$, Enrong Mao ${ }^{2}$, Bo Cheng ${ }^{1, *}$ \\ ${ }^{1}$ Department of Automotive Engineering, Tsinghua University, Beijing, P. R. China 100084 \\ ${ }^{2}$ College of Engineering, China Agricultural University, Beijing, P. R. China 100083 \\ * Corresponding author, Address: Department of Automotive Engineering, Tsinghua \\ University, Beijing, P. R. China, Tel: +86-10-62780355, Fax: +86-10-62780355, Email: \\ chengbo@tsinghua.edu.cn
}

Abstract: The similarity of varied vehicle package is a critical design feature that affects method selection, optimized design and driver performance. However there is limited understanding of what constitutes similarity in package design and limited computer-based support to identify this feature in a layout model. This paper contributes a case-based framework for representing and reasoning about layout similarity that builds on domain-specific ontological modeling and case-based reasoning techniques. Validation study of the system provides evidence that the framework is general and enables a more efficient package layout design process.

Keywords: vehicle package, $\mathrm{CAD}$, ontology, case-based reasoning

\section{INTRODUCTION}

Designers of workplaces and products have three major tasks (Feyen et al. 2000): one, integrating information about processes, tools, machines, parts, tasks, and human operators; two, satisfying design constraints which often conflict; and three, generating a design acceptable to all parties involved. However, while completing these tasks, designers often have difficulty incorporating ergonomics information about the human operator into their designs. Although such information exists for use in the job design process,

Please use the following format when citing this chapter:

Jin, X., Mao, E. and Cheng, B., 2009, in IFIP International Federation for Information Processing, Volume 294, Computer and Computing Technologies in Agriculture II, Volume 2, eds. D. Li, Z. Chunjiang, (Boston: Springer), pp. 1451-1460. 
one reason for the difficulties in using this information is that it is often poorly presented for use by designers.

The layout of the driver workstation is referred to as the vehicle package. Complete definitions of interior points can be found in SAE J1100 and associated practices (SAE 2001). Vehicle occupant packaging is the process of laying out the interior of a vehicle to achieve the desired levels of accommodation, comfort, and safety for the occupants. Creating a vehicle package should take the ergonomic factors into consideration, which include the requirements of driver seating comfort, operating convenience, visibility, etc. Theoretically there might be numerous layout design solutions. However, the following reasons have to be considered: (a) The vehicle cab interior design technology is quite mature and a lot of typical package layout designs have been created and proved to be feasible in iterative practice in different series of vehicle models; (b) Unproved novel design is unlikely to be adopted in fact due to the demands of high cost and high reliability; and (c) Most designers prefer reuse of previous designs as much as possible to reduce the workload because of large number of complicated design tasks and generally restricted time. Owing to these facts, the vehicle package layout design is usually achieved by referring to former design cases.

As one of AI technologies, case-based reasoning offers some advantages compared to other knowledge representation and reasoning formalisms. Cases represent specific knowledge of the domain, are natural and usually easy to obtain (Avramenko 2006). Case-based reasoning has been utilized to solve mechanical design problems since it well reflects the commonly used design methodology of consulting previous designs (designers rarely design an artifact from scratch). Structure-behavior function (SBF) device models are used to represent and comprehend specific design cases, and also to provide necessary knowledge for modifying a retrieved case to fit a new design problem (Han and Lee 2006). Some studies (Chen et al. 2006) applied the CBR for an automotive body assembly process design system in searching the identifying features. Some (Dan 1997) had proposed the framework of EDKBES (Ergonomic design knowledge base expert system) for vehicle interior design combining CAD with expert system technologies. Also RAMSIS was used for interior layout design (Vogt 2005). It worked effectively with a few components and small layout areas. However, serious errors occurred when it performed relatively complicated design tasks. In general, while AI technologies are widely applied in the fields of conceptual design, diagnostic and fault detection, little work has been done to automate and/or support the vehicle package design tasks with the ergonomic analysis.

Contemporary AI technologies can be used to represent knowledge and model reasoning processes. A system for intelligent design of interior layout can be developed on the basis of these technologies. Development of such system is a promising research field. In this paper, a case-based framework for vehicle package design was presented with the ontology employed in the 
development of knowledge representation model, which was a promising way to increase the quality and efficiency of vehicle interior ergonomic design. A coach package problem was selected as a test domain of layout design and analysis because of their importance and frequent interaction in driving practice.

\section{ARCHITECTURE OF THE SYSTEM}

To understand the subtleties for how practitioners think about the design in the context of assessing layout similarity, some researches have been performed to extract the human factor rules and principles (Jin et al. 2005), and approaches of quantifying the degree of comfort have been proposed.

Fig.1 graphically represents the framework that was developed for representing and reasoning about layout similarity:

a) Represent layout similarity: Designers specify their knowledge for defining layout similarity in a computer-interpretable template that is based on the ontology (Fig.1(1)). Users define layout similarity specifications according to their preferences. The system represents the instances of this feature generically, independent of a particular project. This projectindependent knowledge is then utilized to compute similarity when the practitioner is ready to create an outcome for a domain-specific 3D design.

b) Identify layout similarity: A specific package configuration of layout similarity was created based on the practitioner's generic preferences defined in the previous research. Then the formal methods deduce out the geometric, topological, and symbolic similarities between components in the input 3D model and quantify the degree of similarity (Fig.1(2)). The result is a specific package configuration of layout similarity customized for the user.

\section{KNOWLEDGE REPRESENTATION MODEL}

According to the characteristics of layout design, analysis of the following types of knowledge was to be represented within the knowledge representation model:

a) Attributes of the vehicle package layout including: Vehicle types, Component parts, Properties of the layout and its parts, and Spatial and functional relations between component parts within a layout.

b) Human factor problems including: Types of design rules and layout constraints, Properties of the layout ergonomic problems.

c) Knowledge about solution procedures, including: Problem types, Algorithms and their parameters. 

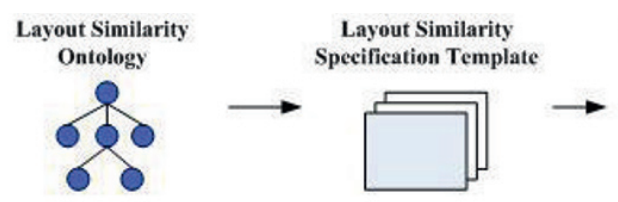

(1) Layout Similarity

Representation

3D HMI layout model

(1) Identify relevant components and attributes

(2) Evaluate similarity between components and between layouts (3) Create groupings and quantify degree of similarity

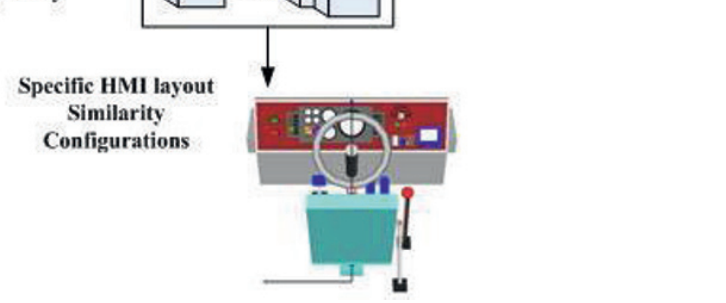

Fig.1: Framework for (1) representing and (2) reasoning about layout similarity to create specific package configurations of layout similarity

d) Dependencies between human factor problem types and properties and the layout design solutions.

e)Cases: a case's description includes definitions of: Physical components, Human factors problem set for this layout, Formulation of a corresponding layout reference, and Saved numerical solution routine specification.

Upon the comparative analysis, domain-specific formal ontology was selected as the knowledge representation model type. Knowledge representation model was developed which comprised: (a) ontology of whole package layout, (b) ontology of human factor principles, (c) ontology of solution techniques, (d) special concepts - subsets of the 'rule' concept, which represented rules and constraints reflecting dependencies between properties of ergonomic problems, layout and solutions.

So, the ontology-based domain knowledge representation model $O B M$ can be structured according to the "human factor problems-layout-solution routine" reasoning schema in the following way: $O B M=\{H P, L P, S P, D E\}$, where $H P$ is the set of elements of description of human factor problems, $L P$ the set of elements of components and layout problems, $S P$ the set of elements of solution procedure descriptions and $D E$ the set of dependencies between properties of ergonomic problems, layout problems and solutions.

This work aims to develop the general structure of the model so that it can be populated by a variety of ergonomic experts and be broadly applicable across a variety of vehicle designs and domains. The domain-specific ontology that was developed to represent layout similarity allowed designers to specify their varied preferences for what component properties needed to 
be similar and how much variation was acceptable for layout similarity to exist. Features were classified into the following types: a) Component Features were features that resulted from components in a $3 \mathrm{D}$ vehicle interior layout model; b) Spacial Features were features that resulted from the spacial relations and functions between components; and c) Macro Features were features that resulted from pre-specified combinations of other features. Layout similarity was a specific class of macro feature.

Fig.2 showed the features and attributes currently represented in the ontology. Each component feature represented the basic attributes of the components using the 'feature set' and 'property set'. Each spacial feature represented the emphasis of what special relation and function of the component would affect a component's location layout using the 'property set' attribute. Each macro feature represented the elements for defining layout similarity in terms of the component properties that need to be similar and the amount of variation that was allowed to exist according to the ergonomic rules. The attributes of the three feature types enabled the designers to represent their varied design preferences based on the layout similarity, and specifying their interior layout design characteristics by highlighting certain part of them.

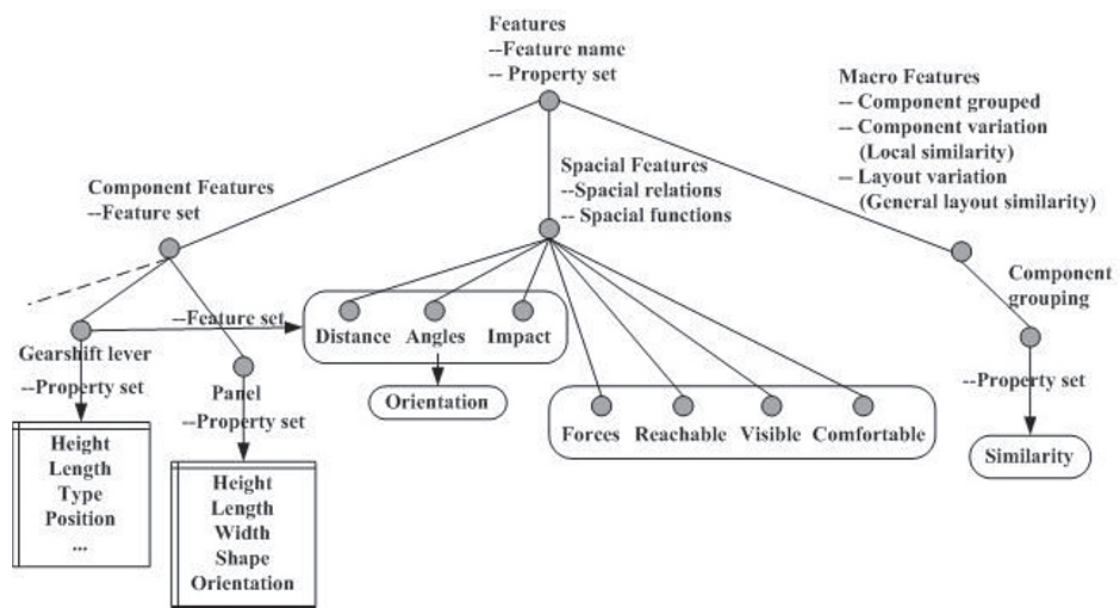

Fig.2: Domain-specific ontology that represented the attributes of package components

The attributes currently formalized in the ontology were to characterize the feature layout similarity. The attributes provided a formal way to specify the different types of component properties (e.g., geometric, symbolic and relational attributes) to be evaluated. The ontology also provided a way to characterize the degree of similarity at the component level (e.g., SeatHeight $\pm 10 \mathrm{~cm}$ ) and at the system level (e.g., 0.1 variation). 
The classification of component attributes facilitated the evaluation of layout similarity. Table1. showed the different component attributes currently implemented in the ontology based on the different case studies conducted to date. The component attributes listed were either represented explicitly in a 3D model or could be derived from a 3D model.

Table 1. Component attributes in the current ontology

\begin{tabular}{|c|c|c|c|c|c|c|c|}
\hline \multirow[t]{2}{*}{ Component } & \multicolumn{3}{|c|}{ Component attributes } & \multirow[t]{2}{*}{ Component } & \multicolumn{3}{|c|}{ Component attributes } \\
\hline & Geometric & Symbolic & Relational & & Geometric & Symbolic & Relational \\
\hline \multirow[t]{6}{*}{ Gearshift } & Height & Type & Reachable & Panel & Height & Type & Visible \\
\hline & Length & Shape & Comfortable & & Length & Curvature & Reachable \\
\hline & PositionX & & Max force & & Width & Shape & \\
\hline & PositionY & & & & PositionX & Orientation & \\
\hline & PositionZ & & & & PositionY & & \\
\hline & & & & & PositionZ & & \\
\hline
\end{tabular}

The overall variation of the components allowed achieving layout similarity as a function of a maximum and minimum range. In the package cases, a minimum of 0.85 and a maximum of 1 were specified to represent an 'ideal' degree range of similarity for the similar case. The component variation corresponded to the local similarity and the layout variation to the overall similarity, both of which were referred as a function of $[0,1]$. When evaluating the overall variation of all the layout components design, designers also require some allowance for specifying degrees of similarity.

\section{REASONING MECHANISM}

CBR is successfully applied to the wide range of problems in various domains. The key tasks of the CBR process are: 1) Query formulation in terms of the knowledge representation model, 2) Retrieval of cases which are the most similar to the query, 3) Adaptation of the selected cases to match the query and 4) Cases retention in the knowledge base (learning of the system).

The performed research had shown that existing CBR algorithms could not be directly applied to ergonomic solutions of package design problems, because this domain had some specific properties: layout problem (case) description was, as a rule, of complex structure, which could vary depending on the technical object and the problem type; values of qualitative and quantitative parameters are to be evaluated taking into account the context (values of other parameters).

Consequently, the following algorithms were developed to operate on the suggested domain model $O B M$ in the framework of the CBR mechanism implementation: CBR-query formulation support algorithm; case retrieval 
algorithm which uses concept-based similarity (CBS) computation algorithm and slot-based similarity (SBS) computation algorithm; case adaptation algorithm, which used ergonomic rules and similarity paths.

The case retrieval algorithm, which was the key part of the CBR reasoner, was based on the similarity measure Sim. As the case indexes were represented in the formal ontology by features, the similarity of cases was reduced to the similarity between the features i1 and i2 of the ontology, which was local similarity. It could be represented as

$$
\operatorname{Sim}\left(i_{1}, i_{2}\right)=\operatorname{Func}\left(C B S\left(i_{1}, i_{2}\right), \operatorname{SBS}\left(i_{1}, i_{2}\right)\right)
$$

where $\operatorname{Sim}$ is the overall similarity of the individuals, $C B S$ the conceptbased similarity, $S B S$ the slot-based similarity, Func a real-valued composition function. The suggested case retrieval algorithm did not calculate the full Sim value for all the cases in the knowledge base. For most cases only the CBS value was calculated, which was much simpler to compute than the SBS, then the maximal possible value Sm of Sim for the given CBS was estimated, and SBS was computed only when $\mathrm{Sm}$ was greater than the current maximal Sim or greater than some given threshold.

The CBS computation algorithm used the vector space model, where each instance was represented by an n-dimensional vector. Components of this vector corresponded to user-defined ontology concepts. If the instance was subsumed by a concept $m$, the corresponding vector component was assigned the appropriate value of the weighting function $W(m)$, if not - zero. CBS was then computed using the well-known cosine measure:

$$
\operatorname{CBS}\left(i_{1}, i_{2}\right)=\frac{\text { Vector }_{1} \text { Vector }_{2}}{\| \text { Vector }_{1}\|\| \text { Vector }_{2} \|}
$$

The SBS computation algorithm was based on one-to-one comparison of individuals' relations, which could represent their parameters and/or structural relations. The SBS computation process was recursive; it started on the given features $i_{1}$ and $i_{2}$, compares one-to-one all the features related to them by ontology relations, and stopped on features with no relations, for which SBS was not computed. Use of weighting coefficients $W$ for relations allowed flexible tuning of the algorithm and use of role similarity functions (tables) adequately handles ontologies with multiple similar relations:

$$
\operatorname{SBS}\left(i_{1}, i_{2}\right)=\sum_{j 1=0}^{n 1} \sum_{j 2=0}^{n 2} w_{j 1} w_{j 2} \operatorname{LocalSim}\left(R_{j 1}, R_{j 2}\right)
$$

where $n_{1}$ is the number of relations of the individual $i_{1}, n_{2}$ the number of relations of the individual $i_{2}, w_{j 1}$ the weight of relation $j_{1}$ of instance $i_{1}, w_{j 2}$ the weight of relation $j_{2}$ of the individual $i_{2}, R_{j 1}$ the $j_{1}$ th relation of the individual $i_{1}, R_{j 2}$ the $j_{2}$ th relation of the individual $i_{2}$, LocalSim the similarity 
function of the two given relations (local similarity). The general schema of the suggested similarity computation algorithm is presented in Fig.3.
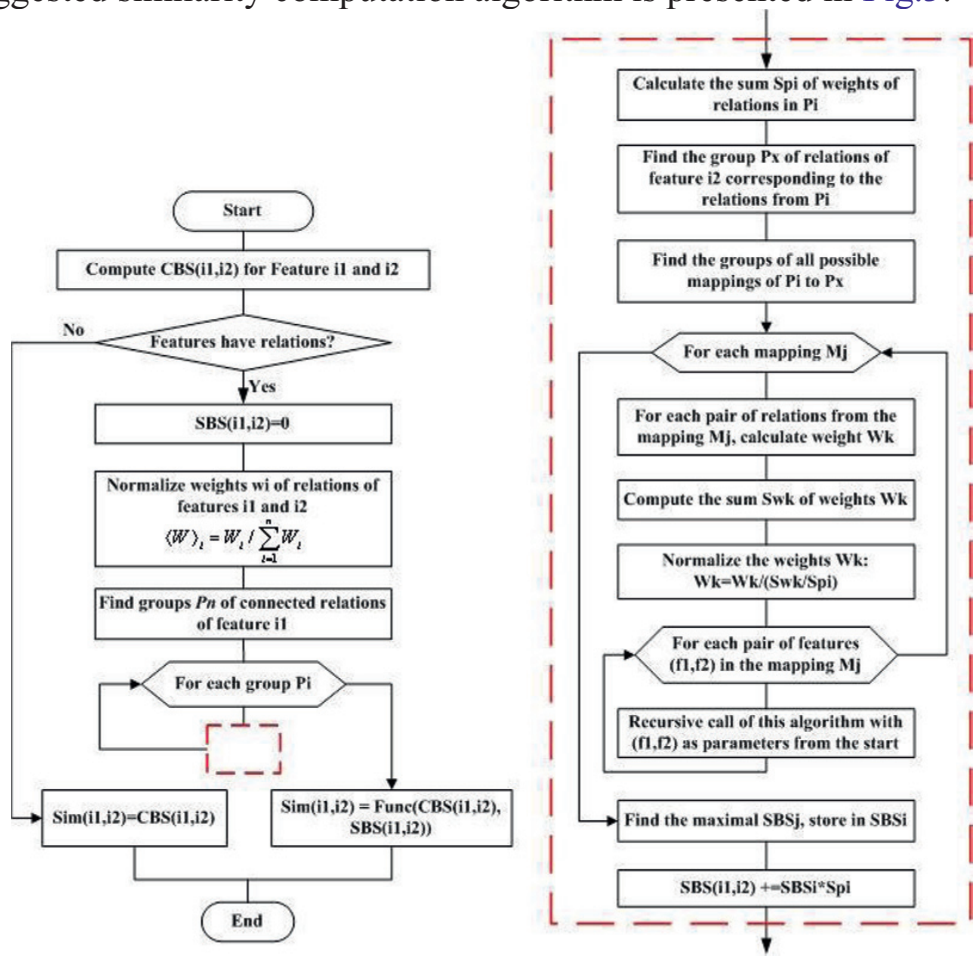

Fig. 3: Overall flowchart of CBS and SBS similarity computation algorithm

\section{CASE STUDY}

Based on the methodologies discussed above, a software tool called Vehicle Package Design Advisor had been developed. In this section, a case study was presented for the design of an automotive panel and instruments that could generate optimum layout considering user preference based on generic ergonomic knowledge base and feasible reasoning mechanisms by using this Advisor.

By the reasoning mechanism proposed above, several similar cases were successfully retrieved, and the basic layout information from the similar cases including the component grouped and detailed information were then displayed when one of the cases was highlighted along with the graphical representations of the components and layout. Fig.4 and Table 2. indicated the results of case retrieval for panel grouped, in which Dist1 was the eye view distance, Dist2the distance from panel to floor, Obliquityl the angle of panel relative to floor, Obliquity2 the eye sight angle relative to the horizontal, Length the effective panel length, Width the effective panel width. 
Based Reasoning
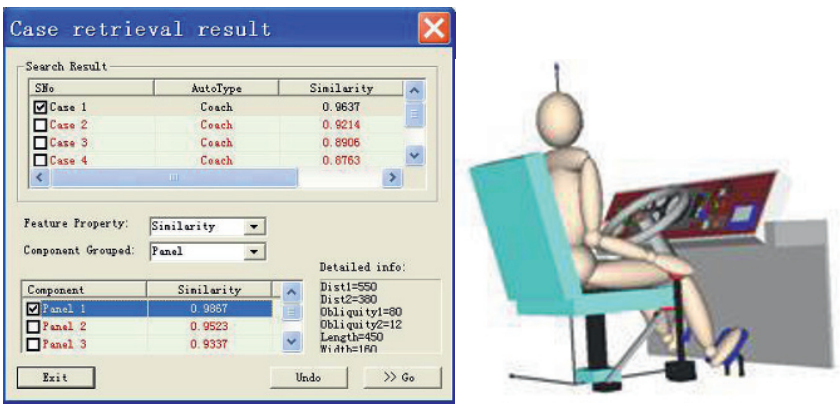

Fig. 4: Results of case retrieval and graphical representations

Table 2. Results of case retrieval for panel grouped

\begin{tabular}{cccccccc}
\hline Items & Dist1 & Dist2 & Obliquity1 & Obliquity2 & Length & Width & Similarity \\
\hline Expectation & $440 \sim 710$ & $350 \sim 450$ & $60^{\circ} \sim 90^{\circ}$ & $10^{\circ}$ & & & \\
Case 1 & 550 & 380 & $80^{\circ}$ & $12^{\circ}$ & 450 & 160 & 0.9867 \\
Case 2 & 600 & 410 & $70^{\circ}$ & $13^{\circ}$ & 480 & 150 & 0.9523 \\
Case 3 & 630 & 440 & $75^{\circ}$ & $10^{\circ}$ & 500 & 180 & 0.9337 \\
\hline
\end{tabular}

After all feasible layout designs were found, the most similar layout case would be lightly revised and evaluated considering seating comfort. Since all the layout cases in this research used certain formerly adopted interior layouts as references, there were possibilities of comparing the manikin seating postures both in the new design and in the referred layout using empirical range of comfort angles (Judic 1993 and Kolich 2000) of driver joints. The grading evaluation method proposed in literature (Jin 2005) was used to estimate the seating comfort. It could be concluded from Table 3 . that the newly designed coach interior layout was better than the referred layout case regarding the comfort quality for users of $50^{\text {th }}$ percentile.

Table 3. Comparison of joint angles of manikin seating posture

\begin{tabular}{ccccc}
\hline Joint & Design sample & Grading & Referred case & Grading \\
\hline Ankle & $95.7^{\circ}$ & better & $90^{\circ}$ & accepted \\
Knee & $108^{\circ}$ & better & $97^{\circ}$ & accepted \\
Elbow & $89^{\circ}$ & accepted & $87^{\circ}$ & accepted \\
Seat back angle & $21^{\circ}$ & accepted & $15^{\circ}$ & bad \\
\hline
\end{tabular}

\section{CONCLUSION}

This paper contributes a framework for vehicle package design by representing and reasoning about layout similarity that was built on ontological modeling and case-based reasoning techniques. It is presented that assumes use of ergonomic knowledge base. The ontology was 
formalized to represent the interior layout component, the component attributes, and the degree of variation required assessing component similarity, etc. A computer implementation of the ontology enables designers to represent their varied preferences for defining layout similarity generically and consistently. A generic reasoning process was also developed to identify domain-specific instances of layout similarity in a given 3D product model. The reasoning mechanism of the systems implemented "ergonomic problems $<->$ Layout design <-> solutions" schema and was based on the CBR technology. The reasoning process evaluates the geometric, topological, and symbolic similarities between components and layouts and quantifies the degree of similarity.

Automating the detection of domain-specific design features, like layout similarity, has the potential to significantly improve the efficiency of the design process. Project teams could perform what-if analyses on different designs and explore a larger variety of design alternatives to identify the optimal design. Users could provide feedback to designers on the specific features that impacted the degree of comfort. Hence, project teams can adjust the case-based models to develop more comfortable designs in less time.

\section{REFERENCES}

Avramenko Y and Kraslawski A. Similarity concept for case-based design in process engineering, Computers \& Chemical Engineering, 2006, 30: 548-557.

Chen, GL, Zhou, JQ and Cai, W. A framework for an automotive body assembly process design system, Computer-Aided Design, 2006, 38: 531-539.

Dan, MP. Using man modeling CAD system and expert systems for ergonomic vehicle interior design. Proceedings of the 13th Triennial Congress of the International Ergonomics Association, 1997, pp. 80-83.

Feyen R, Liu YL and Chaffin D. Computer-aided ergonomics: a case study of incorporating ergonomics analyses into workplace design, Applied Ergonomics, 2000, 31:291-300.

Han YH and Lee KW. A case-based framework for reuse of previous design concepts in conceptual synthesis of mechanisms, Computers in Industry, 2006, 57: 305-318.

Human Factors and Ergonomics in Manufacturing, 2005, 15(2): 197-212.

Jin XP, Song ZH and Mao ER. Research on the vehicle HMI qualitative and quantitive grading evaluation methods, Proceedings of Man-Machine-Environment System Engineering, 2005, 7: 232-236 (in Chinese).

Judic JM. More Objective Tools for the Integration of Postural Comfort in Automotive Seat Design, SAE Technical paper 930113.

Kolich M. Driver Selected Seat Position: Practical Applications, SAE Technical Paper 200001-0644.

Society of Automotive Engineers. Automotive engineering handbook. Warrendale, PA: Author. 2001.

Vogt C., Mergl C. and Bubb H. Interior Layout Design of Passenger Vehicles with RAMSIS, 